\title{
Rare presentation of levamisole-induced leukoencephalopathy in a pediatric patient: seizure
}

\author{
Cem Paketçi ${ }^{\oplus}$, Derya Okur ${ }^{\oplus}$, Semra Hız ${ }^{\oplus}$, Uluç Yiş ${ }^{\oplus}$ \\ Division of Pediatric Neurology, Department of Pediatrics, Dokuz Eylül University Faculty of Medicine, İmir, Turkey.
}

\begin{abstract}
Background. Levamisole is an imidazole derivative used in the treatment of various cancers, dermatological diseases, and parasitosis. Illegal use of levamisole by mixing it with cocaine in order to increase the psychotropic effects has also increased in recent years. Leukoencephalopathy is one of levamisole's most prominent neurological side effects.

Case. Here we present the clinical, laboratory, imaging findings, treatment, and follow-up information of a 12-year-old girl who presented with seizures due to levamisole, which was prescribed to treat vitiligo.

Conclusion. Levamisole-induced leukoencephalopathy should be considered in the differential diagnosis of demyelinating diseases, the neurotoxic effects of the drug should be well understood, and treatment should be initiated as soon as possible.
\end{abstract}

Key words: levamisole, demyelinating diseases, leukoencephalopathies, seizure, vitiligo, cocaine.

Levamisole is the L-isomer of tetramisole. It was first used as an anthelmintic agent in 1966 and was later used in inflammatory and oncological diseases due to its wide immunomodulatory effects. ${ }^{1}$ Levamisole has also been used to treat many dermatological diseases., ${ }^{2,3}$ It was approved by the United States Food and Drug Administration in 1991 as an adjuvant therapy in the treatment of colorectal cancers. However, over time, various side effects such as agranulocytosis, thrombocytopenia, and leukocytoclastic vasculitis have been observed and it was withdrawn from use. The most notable neurological side effect was multifocal inflammatory leukoencephalopathy (MIL), which was first seen in patients receiving a combination of 5-fluorouracil and levamisole therapy for colon cancer treatment. Since treatment of non-cancer diseases withlevamisole alone also caused leukoencephalopathy, it

Cem Paketçi

paketci@hotmail.com

Received 3rd September 2020, accepted 16th October 2020. was thought to be responsible for causing this side effect. ${ }^{4}$ Due to all these side effects, the pharmacological use of levamisole in humans has become very limited. However, in recent years, levamisole has been illegally used to increase the psychotropic effect of cocaine. Sixty-five percent of cocaine worldwide is contaminated with levamisole and it has been shown that leukoencephalopathy developing in individuals using cocaine is due to levamisole. ${ }^{5}$ Neurological symptoms such as altered consciousness, ataxia, aphasia, hemiparesis, quadriplegia, seizure, and facial palsy that manifest as a result of leukoencephalopathy can be treated with steroids, immunoglobulin and plasmapheresis treatments. ${ }^{4}$ Although there are reports regarding levamisole induced leukoencephalopathy (LIL) in the literature, most recent studies have focused on leukoencephalopathy that develops in cocaine users. As far as we know, there is only one study that reported two pediatric patients with LIL. ${ }^{6}$ Here, we present a case of a 12-year-old patient that developed LIL. 


\section{Case Report}

A 12-year-old girl, who did not report any previously known diseases, presented with generalized tonic-clonic seizures. It was learned that the patient had previously fainted a week ago and since brain tomography and electroencephalography (EEG) examination performed at that time were normal, the patient was diagnosed with syncope. The patient, who was born at 38 weeks by Caesarean section and weighed $2840 \mathrm{~g}$, did not have any problems during the postnatal period. She reached all her developmental milestones at appropriate ages and her family history was unremarkable. The patient had a 6.5-year-old healthy sister. The patient had recently experienced emotional lability, light-headedness and mild headache. The physical examination was normal, except for hypopigmentation in the right lower quadrant of the abdomen. When questioned, it was learned that the patient was started on oral levamisole treatment 6 weeks ago due to vitiligo, and that she had taken a total of $720 \mathrm{mg}$ levamisole.

Although repeated one-hour sleep and wakefulness EEG examination was found to be normal, levetiracetam treatment was started, since the patient had experienced two episodes within one week. Brain MRI revealed T2 and FLAIR hyperintensities in the bilateral periventricular regions and right anterior section of centrum semiovale. Some of these lesions showed gadolinium enhancement, one lesion in the right frontal region was shaped like a ring (Fig. 1A-C).

No cells were seen in the patient's cerebrospinal fluid (CSF) and her biochemistry results were normal. CSF cultures were sterile. The CSF viral serology panel that included HSV, adenovirus, enterovirus and CMV was negative. The patient received $1 \times 1 \mathrm{gr}$ pulse methylprednisolone treatment for three days and her CSF oligoclonal band was negative and IgG index was 0.49 (n: $<0.7)$. The serology results of TORCH, hepatitis,

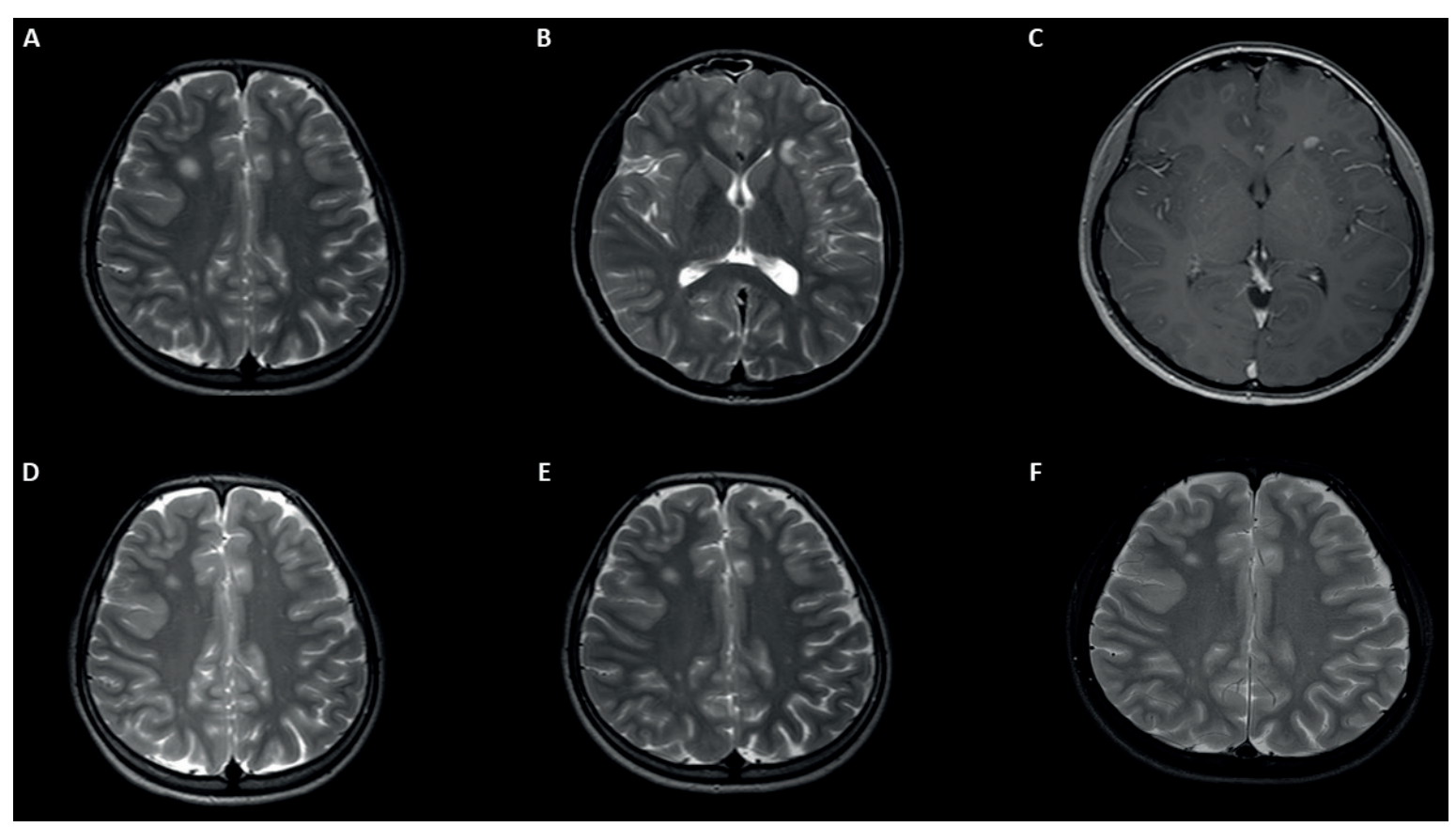

Fig. 1. A-C. MR images at admission. Axial T2-weighted images showed white matter lesions in both cerebral hemispheres (A, B); Axial T1-weighted images after gadolinium administration demonstrated enhancements and also ring enhancement of right frontal subcortical lesion (C). D-F. Axial T2-weighted images at one month, one year and 2,5 years later (respectively), lesions were significantly decreased and contrast enhancement after gadolinium administration had disappeared. There was no new lesion. 
lyme and mycoplasma were negative. Moreover, serum neuromyelitis optica and myelin oligodendrocyte glycoprotein antibodies were negative. The brain MR angiography performed for the differential diagnosis of vasculitis was found to be normal and the patient's erythrocyte sedimentation rate was $4 \mathrm{~mm} / \mathrm{h}$ (n: 0-20). Antinuclear antibody, anti-ds DNA, anti-cardiolipin, and lupus anticoagulant were negative. Since the patient did not have any abnormal test results, the white matter lesions were thought to be associated with levamisole treatment. Oral methylprednisolone treatment was reduced and discontinued in one month.

No clinical complaints were observed during the 2.5-year follow-up. No new lesions were detected in brain MRI examinations and regression was observed in existing lesions over time (Fig. 1D-F). The patient, whose levetiracetam treatment was discontinued, continues to be followed without treatment. Informed consent was received from the family.

\section{Discussion}

Levamisole, which is a synthetic antihelmintic drug, triggers cellular immunity by changing the balance of T helper- 1 cell / T helper-2. While it increases the levels of some interleukins (IL-2 and IL-12) it causes others to decrease (IL-4, IL5, and IL-10). Its toxic effects are generally mild. Common side effects are gastrointestinal disorders, skin manifestations, and hematological disorders. There is a wide potential for neurological side effects such as headache, vertigo, vomiting, aphasia, blurred vision, diplopia, weakness that can be seen in $1.3-5 \%$ of patients. ${ }^{3}$

LIL is a delayed demyelinating leukoencephalopathy. ${ }^{7}$ Although the cause has not been fully revealed, it has been reported that in dogs treated with levamisole perivascular areas in the brain and meninges are surrounded by mononuclear cells. Levamisole has not been shown to cause inflammation or demyelination in mice, therefore it is thought that it does not have a toxic effect on myelin. However, administration of levamisole increased inflammation and demyelination in mice infected with a virus that can cause demyelination. Therefore, levamisole is thought to cause leukoencephalopathy by increasing inflammation that occurs against the presence of a symptom-free antigen. ${ }^{3}$ LIL has been reported in patients receiving cancer treatments or individuals using levamisole for anthelmintic purposes, however recently there has been a surge of LIL cases in cocaine users. There are only two pediatric LIL cases reported in the literature. ${ }^{6}$

LIL usually occurs subacutely within the first two months of levamisole use and cognitive, motor and less frequently sensory clinical findings (ataxia, consciousness change, hemiparesis / quadriplegia, facial palsy, diplopia, dysarthria) can be seen, while seizures are rare. ${ }^{7}$ In our patient, the symptoms were seen in the subacute period, 6 weeks after the use of levamisole. Interestingly, our patient's most prominent neurological finding was a seizure. Apart from our patient, seizures have been reported in only four patients in the literature: in two adults that used levamisole as an anthelmintic due to ascaris, in another adult that used it due to colon cancer, and in one adolescent patient. ${ }^{6-8}$ The adolescent patient experienced epilepsia partialis continua due to the use of levamisole and was treated with levetiracetam. ${ }^{8}$

LIL has many similarities with acute disseminated encephalomyelitis (ADEM) and multiple sclerosis (MS). The diagnosis of LIL is made clinically, while CSF examinations, EEG and MRI are ancillary studies. Levamisole has been reported to cause ADEM, where it develops acutely or subacutely following a febrile illness. Inflammation findings are expected in CSF analysis and serological examinations. Although EEG abnormalities are common, they are nonspecific. Diffuse slowing, which mostly refers to cortical dysfunction, are observed. ${ }^{7}$ Our patient did not have a febrile infection history, there were no signs of inflammation and serological 
examinations were normal. EEG examination was normal at sleep and wakefulness. Since rheumatological tests and MR angiography were normal, vasculitis was ruled out.

The MR images of LIL patients often indicate multifocal demyelinating changes in subcortical white matter and periventricular areas. Frontal and parietal lobes are affected more often. Ringlike enhancement of the lesions is typical. ${ }^{6}$ It may not be easy to differentiate these LIL MRI findings from MS and ADEM. However, unlike MS and ADEM, gray matter involvements such as basal ganglia, thalamus and brainstem are not expected, and the corpus callosum is generally preserved. ${ }^{7}$ In the MR examination of our patient, demyelinating lesions, some with circular enhancement, were observed in the subcortical white matter and periventricular areas in frontal and parietal lobes of both hemispheres, while the gray matter involvement was not observed.

Levamisole treatment is used in many different dermatological diseases such as verruca, aphthous ulcers, pemphigus, erythema multiforme, and vitiligo. ${ }^{2}$ In LIL, the symptoms and lesions appear independent of the levamisole dose, which indicates that its toxic effect is idiosyncratic. ${ }^{3}$ Although symptoms are most commonly seen as subacute within 8 weeks of use, there have been cases where symptoms began 1 day or months later. The symptoms can be seen after months of chronic use, or after a single dose of $50 \mathrm{mg}$. ${ }^{6,7}$ As with most cases presented in the literature, in our case the symptoms appeared in the sixth week with a total of $720 \mathrm{mg}$ levamisole use. The treatment of LIL is aimed at suppressing the immune response and good long-term results have been reported with steroids, IVIg, and plasmapheresis treatments. Permanent problems such as ataxia and quadriplegia may be encountered if the treatment is delayed or neglected. ${ }^{7,9}$ No recurrence of seizures was observed in our patient after steroid treatment, and the imaging findings improved after one month. In the subsequent 2.5-year follow-up, no clinical findings have developed, patient's lesions have significantly regressed and no new lesions were observed.

In conclusion, the neurotoxic effects of levamisole can occur regardless of the dose. Patients that have taken levamisole may present with seizures and their encephalopathy symptoms may be mild. If possible, we suggest avoiding the use of levamisole for the treatment of dermatological diseases.

\section{REFERENCES}

1. Amery WK, Bruynseels JP. Levamisole, the story and the lessons. Int J Immunopharmacol 1992; 14: 481-486.

2. Scheinfeld N, Rosenberg JD, Weinberg JM. Levamisole in dermatology : a review. Am J Clin Dermatol 2004; 5: 97-104.

3. Cheng YC, Po HL. Leukoencephalopathy after levamisole for the treatment of verrucae. Acta Neurol Taiwan 2011; 20: 262-266.

4. Larocque A, Hoffman RS. Levamisole in cocaine: unexpected news from an old acquaintance. Clin Toxicol (Phila) 2012; 50: 231-241.

5. Brunt TM, van den Berg J, Pennings E, Venhuis B. Adverse effects of levamisole in cocaine users: a review and risk assessment. Arch Toxicol 2017; 91: 2303-2313.

6. Xu N, Zhou W, Li S, Zhou G, Zhang N, Liang J. Clinical and MRI characteristics of levamisoleinduced leukoencephalopathy in 16 patients. J Neuroimaging 2009; 19: 326-331.

7. WuVC,HuangJW, LienHC, etal.Levamisole-induced multifocal inflammatory leukoencephalopathy: clinical characteristics, outcome, and impact of treatment in 31 patients. Medicine (Baltimore) 2006; 85: 203-213.

8. Aberastury MN, Silva WH, Vaccarezza MM, Maxit C, Agosta G. Epilepsia partialis continua associated with levamisole. Pediatr Neurol 2011; 44: 385-388.

9. Yan $\mathrm{R}, \mathrm{Wu} \mathrm{Q}$, Ren J, et al. Clinical features and magnetic resonance image analysis of 15 cases of demyelinating leukoencephalopathy induced by levamisole. Exp Ther Med 2013; 6: 71-74. 